\section{Every kind of edema is lymphedema}

\section{Vaughan Keeley \\ Consultant in Lymphedema, Derby \\ Teaching Hospitals, Derby, and Hon. \\ Prof. University of Nottingham, UK}

\section{Introduction}

Lymphedema can be defined as tissue swelling due to a failure of lymphatic drainage. For many healthcare professionals it is commonly considered to arise from lymphatic damage due to cancer treatment (secondary lymphedema) or as a genetic dysplasia of the lymphatic system (primary lymphedema). However, in clinical practice, chronic swelling may be due to a number of causes including venous disease, immobility, chronic heart failure, obesity and drugs.

To cover this wide range of causes, the term chronic edema was coined to create a standardised definition for use in prevalence studies. ${ }^{1}$

Chronic edema is a broad term used to describe edema which has been present for more than three months and involves one or more of the following areas: limbs, hands/feet, upper body (breast/chest wall, shoulder, back), lower body (buttocks, abdomen), genital (scrotum, penis, vulva), head, neck or face.

This term covers what would have been conventionally considered to be lymphedema but also includes swelling where a lymphatic abnormality is not the main underlying cause.

However, in the literature there is confusion about the use of this definition and, sometimes, chronic edema and lymphedema are described as two separate entities.

\section{The pathophysiology of periph- eral edema}

All peripheral edema is due to an accumulation of fluid in the interstitial space. This arises when the amount of fluid passing into the interstitial space exceeds that which is drained away.

\section{Capillary filtration}

The traditional Starling model of tissue fluid formation proposed that fluid flowed out of capillaries into the tissues as a result of the net outcome of opposing pressures across the capillary wall (capillary filtration). These pressure gradients are the hydrostatic pressure gradient due to the difference in physical pressure inside the capillary compared with that in the interstitial space and the colloid osmotic pressure gradient arising from the attraction of water by proteins due to different protein concentrations in the interstitial space and within the capillary. Capillary filtration was also determined by the permeability of the capillary wall.

By applying estimates of the pressure gradients available at the time of the original work, the model suggested that there was an outflow of fluid from the arteriolar end of the capillary and $90 \%$ of this was reabsorbed into the venous end of the capillary. The remaining $10 \%$ of fluid, which had been filtered into the tissues, was drained via the lymphatic system.

This was based upon the concept that hydrostatic pressure at the venous end of the capillary was lower than at the arterial end, that protein was concentrated within their capillary along its length due to the relative impermeability of the capillary wall to protein and, therefore, the colloid osmotic pressure gradient at the venous end of the capillary exceeded the hydrostatic pressure gradient resulting in reabsorption of fluid.

A recent revision of this model has led to a significant change in our understanding of how fluid moves into and out of the interstitial space. $^{2}$

Levick and Michel have proposed that the capillary wall functions as a small pore semipermeable membrane, which arises from the endothelial glycocalyx layer where it covers the intercellular junctions and separates the plasma from the sub subglycocalyx space. It is the protein concentration in this sub glycocalyx space, which has an important impact on the balance of the hydrostatic and colloid osmotic pressure gradients in the Starling model.

Based upon this new model, in the steady state in most capillary beds, the balance of the hydrostatic and colloid osmotic pressure gradients results in net filtration of fluid along the whole length of the capillary with no reabsorption at the venous end.

This means that $100 \%$ of the capillary filtrate is taken up by the lymphatic system and raises the importance of the lymphatic system in the maintenance of interstitial fluid homeostasis.

\section{Lymphatic drainage}

The drainage of fluid from the interstitial space into the initial lymphatics is determined by a hydrostatic pressure gradient. The higher the interstitial pressure, the greater the lymph flow until a transport capacity maximum is reached. ${ }^{3}$ Lymph is transported through the lymphatics mainly
Correspondence: Vaughan Keeley, Consultant in Lymphedema, Derby Teaching Hospitals, Derby, and Hon. Prof. University of Nottingham, UK

E-mail: vaughan.keeley@nhs.net

Conference presentation: International Compression Club (ICC) Meeting, Rotterdam, 2018.

This work is licensed under a Creative Commons Attribution 4.0 License (by-nc 4.0).

(C) Copyright V. Keeley, 2018

Licensee PAGEPress, Italy

Veins and Lymphatics 2018; 7:7992

doi:10.4081/vl.2018.7992

by contractions of smooth muscle in their walls (lymphangions) with the direction of flow determined by valves.

\section{Edema formation}

Edema therefore arises when capillary filtration exceeds lymphatic drainage.

If lymphatic drainage is impaired and capillary filtration is normal, then edema will develop (conventional lymphedema).

If there is an increase in capillary filtration e.g. due to venous hypertension, then lymphatic drainage will increase until it reaches its maximum transport capacity when edema will occur. Edema only occurs when the lymphatic system fails to deal with the increased capillary filtration and, therefore, can be considered to be lymphedema.

In situations of persistent increased capillary filtration and lymphatic drainage, over time the lymphatics become damaged and flow reduces, resulting in a conventionally understood secondary lymphedema.

Therefore, all edema can be considered to be lymphedema. ${ }^{4}$

\section{Conclusions}

In clinical practice, however, all types of chronic edema do not have the same underlying abnormality and there are different effects on the tissues e.g. lipodermatosclerosis is seen in chronic venous disease and adipose tissue deposition where the underlying problem is lymph stasis.

Chronic edema can arise when the underlying abnormality is a failure of lymphatic drainage e.g. primary lymphedema and lymphedema secondary to cancer treatments.

It can also arise from situations where the primary abnormality is an increased capillary filtration rate such as in venous 
hypertension, heart failure, immobility (due to failure of the calf muscle pump leading to increased venous pressure), and hypoalbuminaemia (e.g. in advanced cancer).

In summary, all chronic edema could be termed lymphedema (primary or secondary). However, in clinical assessment, it is helpful to understand the underlying cause or causes in order to determine the most appropriate treatment and consider the best method of preventing progression e.g. man- aging venous hypertension may prevent secondary damage to the lymphatic system.

\section{References}

1. Moffatt CJ, Franks PJ, Doherty DC, et al. Lympho: an underestimated health problem. QJM 2003;96:731-8.

2. Levick JR, Michel CC. Microvascular fluid exchange and the revised Starling principle. Cardiovasc Res 2010;87:198210.

3. Scallan J, Huxley VH, Korthuis RJ. Capillary fluid exchange: regulation, functions and pathology. Morgan and Claypool Life Sciences; 2010.

4. Mortimer PS, Rockson SG. New developments in clinical aspects of lymphatic disease. J Clin Invest 2014;124:915-21. 